Primary mode changes due to longitudinal wall oscillation in two-dimensional channel flow

This content has been downloaded from IOPscience. Please scroll down to see the full text. 2014 Fluid Dyn. Res. 46025502

(http://iopscience.iop.org/1873-7005/46/2/025502)

View the table of contents for this issue, or go to the journal homepage for more

Download details:

IP Address: 133.56.199.32

This content was downloaded on 20/08/2014 at 01:58

Please note that terms and conditions apply. 


\title{
Primary mode changes due to longitudinal wall oscillation in two-dimensional channel flow
}

\author{
Takashi Atobe \\ Japan Aerospace Exploration Agency, Jindaiji-Higashi, Chofu, Tokyo 182-8522, Japan \\ E-mail: atobe.takashi@jaxa.jp
}

Received 4 April 2013

Accepted for publication 7 January 2014

Published 5 February 2014

Communicated by M Asai

\begin{abstract}
The stabilizing effect of longitudinal wall oscillation on two-dimensional channel flow is analytically investigated. Because the flow field has temporal periodicity, stability can be described by the Floquet theory. To do so, a monodromy matrix is derived from a time-dependent Orr-Sommerfeld equation using the collocation method, and then stability is determined by the use of the Floquet exponents. Systems of this kind are governed by the frequency and amplitude of wall oscillation and by the Reynolds number $R_{e}$, which is determined by the maximum velocity of flow and half the distance between two walls. For Reynolds numbers in the range 6000-10 000, there is a region where a stable Tollmein-Schlichting (TS) mode appears in the parameter space. When the amplitude of wall oscillation increases, the disturbance wave is generally stabilized. This effect is large, especially for the TS mode. Consequently, the primary mode of unstable disturbance is changed from the TS mode to oblique modes.
\end{abstract}

\section{Introduction}

Drag reduction is one of the most important issues for the physical efficiency of public transport systems. For aircraft, the influence of skin friction on total drag is relatively large. The magnitude of skin friction depends on the condition of the boundary layer flow. In general, skin friction is large when the boundary layer flow is turbulent. Thus, from the viewpoint of flow control, our interest is how to delay laminar-turbulent transition and how to decrease turbulent shear stress. 
Many methods have been proposed for achieving the above purpose. The most fundamental solution is to optimize the shapes of objects. Passive flow-control devices, such as 'sharkskin', 'riblets' and microvortex generators, can be effective in practical use. However, a time-periodic flow can be expected to reduce friction drag directly because it seems that flow is stabilized by periodicity.

Flow periodicity is of significant theoretical interest. Many studies have been conducted on time-periodic flows such as the Stokes-boundary layer, plane Poiseuille flow, plane Couette flow and pipe flow. In particular, plane Poiseuille flow, a two-dimensional (2D) channel flow, is known to be a realistic model that yields useful information by its description as an exact solution of a linear equation derived from the Navier-Stokes equation; theoretical and numerical investigations have elucidated its essential features (Orszag 1971, Kim et al 1987). For this reason, various studies aimed at drag reduction use plane Poiseuille flow. As passive controls, wavy walls and rough surfaces have been investigated (Floryan 1997, Selvarajan et al 1999). As active controls, rippling walls, vibrating walls and sucking and blowing walls have been studied (Choi et al 1994, Sumitani and Kasagi 1995, Floryan et al 2002). From the biomechanical viewpoint, pulsatile flow is also studied because it is very important in the blood flow in arteries. Zamir (2000) summarized the fundamentals of the hydrodynamics of the blood flow.

Among the studies of active controls, Jung et al (1992) first demonstrated a reduction in wall shear stress from turbulent channel flows by spanwise wall oscillation. A follow-up study by Quadrio and Ricco (Choi 2002) numerically demonstrated a $44.7 \%$ reduction in friction drag reduction, which corresponds to net energy savings of $7.3 \%$. Flow modified by spanwise wall oscillation not only offers reduced drag but also is analogous to simple coupling of channel flow in the Stokes layer. For these reasons, considerable effort has been devoted to this problem (Quadrio and Ricco 2004, Jovanovic 2008, Ricco and Quadrio 2008).

Although this modified channel flow with spanwise wall oscillation can be simplified on the basis of the Stokes layer, its base flow is fundamentally a three-dimensional (3D) flow. Thus, for the purpose of simplification, the present study focuses on the stabilization of the plane Poiseuille flow by longitudinal wall oscillation. Thus, previous studies about walloscillating channel flows will be helpful to review. Grosch and Salwen (1968) showed that flow is stabilized when the amplitude of the wall oscillation is relatively smaller than the mean flow. Herbert (1972) attributed this phenomenon to energy transfer on the wall and concluded that oscillation of the base flow can suppress energy transfer. Hall (1975) used Floquet theory to predict that wall oscillation could act as a stabilizer under a very high frequency condition. Davis (1976) gave a broad survey of the effects of time periodicity on flow stability. Subsequent studies have likewise shown very interesting results. A detailed follow-up to Grosch and Salwen (1968) by Von Kerczek (1982) discovered a small discrepancy in threshold values. Blennerhassett and Bassom (2002) used Floquet theory to discuss the structure of the eigenfunction that links wall velocities. Thomas et al (2011) investigated this type of flow as a perturbation of small channel flow on the Stokes layer and thereby showed that stationary neutral modes do not exist.

The above-mentioned studies offer valuable knowledge, but the determined critical values of stability differ among them. In addition, previous studies considered the stability of 2D disturbances. In general, the oblique Tollmein-Schlichting (TS) wave and other 3D waves also play an important role in flow stability. Thus, the present study aims to confirm the structure of stability in the parameter space and investigates the effect of wall oscillation on 3D disturbance waves.

In section 2, the model flow and the formulation of the Floquet analysis using a timedependent Orr-Sommerfeld equation are explained with an idea based on the collocation 


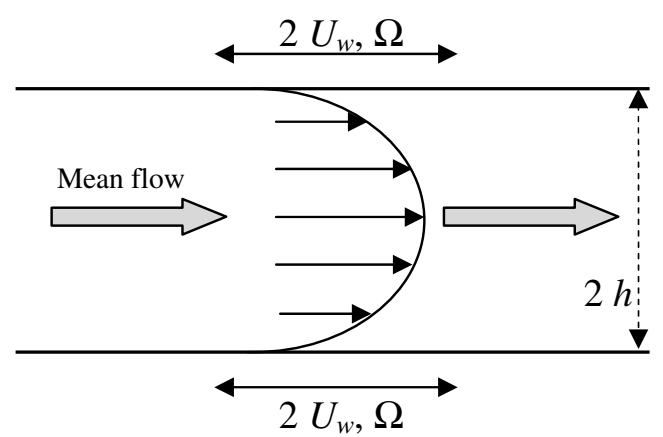

Figure 1. Schematic view of the modified channel flow.

method. In section 3, the stabilizing effects of wall oscillation is discussed by using the Floquet exponents. Primary mode changes are also discussed. Finally, conclusions are given in section 4 .

\section{Formulation of Floquet analysis}

\subsection{Model flow}

Figure 1 shows the flow model under consideration. Here, $\Omega$ and $U_{\mathrm{w}}$ are the frequency and amplitude of the longitudinal wall oscillation, respectively. Thus, this system is described by $\Omega, U_{\mathrm{w}}$ and the Reynolds number. The Reynolds number $R_{e}$ is defined as $h \cdot U_{\max } v^{-1}$, where $U_{\max }$ is the maximum velocity of the mean flow in the absence of wall oscillation, $v$ is the kinematic viscosity and $h$ is half the distance between two walls.

The coordinate system $(x, y, z)$ corresponding to the physical space is adopted, where $x$ is the streamwise direction, $y$ is the direction normal to the wall and $z$ is the spanwise direction. Because the Stokes layer is also an exact solution of a linear equation derived from the Navier-Stokes equation and the plane Poiseuille flow can be likewise described, this modified flow can be described as a superposition of those two exact solutions. From this description, linear stability analysis using Floquet theory is applied with the collocation method. Formally, the modified flow considered here is a superposition of the exact solutions of linear governing equations as follows:

$$
\frac{\partial U}{\partial t}-v\left(\frac{\partial^{2} U}{\partial y^{2}}+\frac{\partial^{2} U}{\partial z^{2}}\right)=-\frac{1}{\rho} \frac{\partial p}{\partial x} .
$$

Here, $\rho$ is the density. This equation is derived from the incompressible Navier-Stokes equation under the parallel flow assumption. In this context, the flow can be represented as $\boldsymbol{U}=\boldsymbol{U}(U(y, t), 0,0)$, where $U(y, t)$ is

$$
U(y, t)=1-y^{2}+U_{\mathrm{w}} \operatorname{Re}\left[\frac{\cosh (k y)}{\cosh (k)}\right] \exp (\mathrm{i} \Omega t) .
$$

Here, $k \equiv \sqrt{\Omega / 2 v}$, and i denotes the imaginary unit. Equation (2) consists of the plane Poiseuille flow and the Stokes layer. In the Floquet analysis, equation (2) is used as the base flow. The velocity profiles calculated by equation (2) for $U_{\mathrm{w}}=0.5$ at each $1 / 8$ period are shown in figure 2 . Because the velocity field is symmetric, only half of the field is shown. 


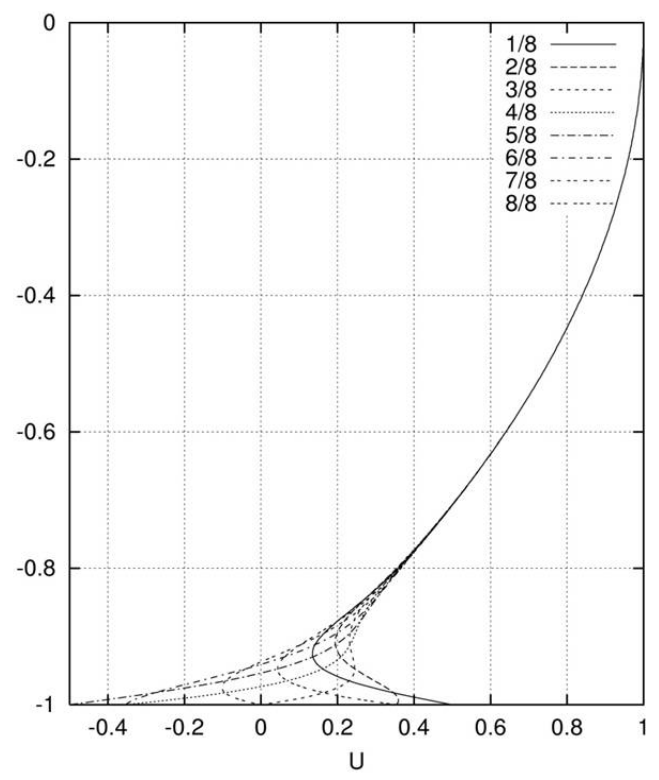

Figure 2. Velocity profile calculated from equation (2).

\subsection{Time dependent Orr-Sommerfeld equation}

When the flow field is described by the base flow $\boldsymbol{U}$ and small disturbances $\boldsymbol{u}^{\prime}$ and $p^{\prime}$, the linearized disturbance equation for $\boldsymbol{u}^{\prime}$ can be derived from the Navier-Stokes equation as follows:

$$
\frac{\partial \boldsymbol{u}^{\prime}}{\partial t}+(\boldsymbol{u} \cdot \operatorname{grad}) \boldsymbol{u}^{\prime}+\left(\boldsymbol{u}^{\prime} \cdot \operatorname{grad}\right) \boldsymbol{u}=-\frac{1}{\rho} \operatorname{grad} p^{\prime}+v \nabla^{2} \boldsymbol{u}^{\prime}
$$

Now, we assume that the small disturbance can be described as a modal plane wave

$$
\boldsymbol{u}^{\prime}(x, y, z, t)=\hat{\boldsymbol{u}}(y, t) \exp [\mathrm{i}(\alpha x+\gamma z)]
$$

where $\alpha$ and $\gamma$ are real wave numbers in the $x$ and $z$ directions, respectively. Substituting equation (4) into (3) with the equation of continuity, we obtain a time-dependent Orr-Sommerfeld equation, which takes the form

$$
\left[\left(\frac{\partial}{\partial t}+\mathrm{i} \alpha U(y, t)\right)\left(D^{2}-\alpha^{2}-\gamma^{2}\right)-\mathrm{i} \alpha D^{2} U(y, t)\right] \hat{v}(y, t)=\frac{1}{R}\left(D^{2}-\alpha^{2}-\gamma^{2}\right)^{2} \hat{v}(y, t),
$$

where $D$ is the differential operator with respect to $y$.

\subsection{Floquet exponents}

If equation (5) can be rewritten in the form

$$
\frac{\partial}{\partial t} \hat{v}(y, t)=G(y, t) \hat{v}(y, t)
$$


because of the periodicity of function $G$, we can expect from Floquet theory that the solution of equation (6) can be described as

$$
\hat{v}_{i}(y, t)=\mathrm{e}^{\mu_{i} t} \varphi_{i}(y, t) \quad(i=1,2, \ldots) .
$$

Here, $\phi_{i}(y, t)$ is a periodic function with period $T$ and $\mu_{i}$ is a complex number, called a Floquet exponent in this context. If the real part of $\mu_{i}$ is positive, then the system will be unstable.

Thus, in order to rewrite equation (5)in the form of equation (6), the Chebyshev spectral collocation method is employed. For this, Gauss-Lobatto scheme (Canuto et al 1988) is adopted for the collocation points

$$
y_{j}=\cos \frac{\pi j}{N+1} \quad(j=0,1,2, \ldots, N) .
$$

Then, equation (5) can be rewritten as

$$
\left(D_{i j}^{(2)}-\alpha^{2}-\gamma^{2}\right) \frac{\mathrm{d}}{\mathrm{d} t}\left(\begin{array}{l}
\hat{v}\left(y_{0}, t\right) \\
\hat{v}\left(y_{1}, t\right) \\
\vdots \\
\hat{v}\left(y_{N}, t\right)
\end{array}\right)=g_{i j}\left(\begin{array}{l}
\hat{v}\left(y_{0}, t\right) \\
\hat{v}\left(y_{1}, t\right) \\
\vdots \\
\hat{v}\left(y_{N}, t\right)
\end{array}\right)
$$

where $D_{i j}^{(2)}$ is the differential matrix of order $(N+1) \times(N+1)$. If the inverse matrix of $\left(D_{i j}^{(2)}-\alpha^{2}-\gamma^{2}\right)$ exists, then equation (9) can be written in the form

$$
\frac{\mathrm{d}}{\mathrm{d} t} \hat{v}\left(y_{j}, t\right)=G_{i j} \hat{v}\left(y_{j}, t\right)
$$

When the function $\hat{v}$ is expanded to $N+1$, equation (9) is written as follows:

$$
\frac{\mathrm{d}}{\mathrm{d} t}\left(\begin{array}{lll}
\hat{v}_{1}\left(y_{0}, t\right) & \ldots & \hat{v}_{N+1}\left(y_{0}, t\right) \\
\hat{v}_{1}\left(y_{1}, t\right) & \ldots & \hat{v}_{N+1}\left(y_{1}, t\right) \\
: & : & : \\
\hat{v}_{1}\left(y_{N}, t\right) & \ldots & \hat{v}_{N+1}\left(y_{N}, t\right)
\end{array}\right)=G_{i j}\left(\begin{array}{lll}
\hat{v}_{1}\left(y_{0}, t\right) & \ldots & \hat{v}_{N+1}\left(y_{0}, t\right) \\
\hat{v}_{1}\left(y_{1}, t\right) & \ldots & \hat{v}_{N+1}\left(y_{1}, t\right) \\
: & : & : \\
\hat{v}_{1}\left(y_{N}, t\right) & \ldots & \hat{v}_{N+1}\left(y_{N}, t\right)
\end{array}\right) .
$$

If equation (11) is simply described as

$$
\frac{\mathrm{d}}{\mathrm{d} t} F(t)=G(t) F(t)
$$

from the Floquet theory, then we can expect that the solution of equation (12) has the form

$$
F(t)=\mathrm{e}^{Q t} \Phi(t)
$$

Here, $\boldsymbol{\Phi}(t)$ is an arbitrary periodic function with period $T, \mathrm{e}^{Q t}$ is a monodromy matrix for $F(t)$ and $Q$ consists of $N+1$ Floquet exponents. Because of the character of the periodic function $\mathbf{\Phi}(t)$

$$
F(0)=\Phi(0) \equiv I .
$$

Thus

$$
F(T)=\mathrm{e}^{Q T} \Phi(T)=\mathrm{e}^{Q T} .
$$




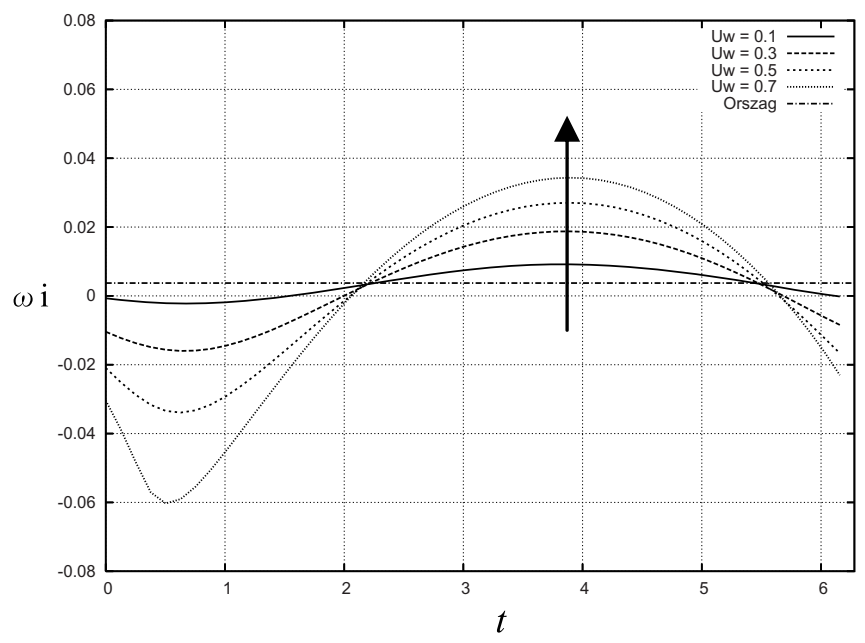

Figure 3. Variation of $\omega_{\mathrm{i}}$ during one period with $R_{e}=10000,(\alpha, \gamma)=(1,0), \Omega=0.1$ and $U_{\mathrm{w}}=0.1,0.3,0.5$ and 0.7 .

Therefore, the Floquet exponents are obtained as

$$
Q=\frac{1}{T} \ln F .
$$

By denoting the eigenvalues of the matrix $Q$ as $\mu$ and the eigenvalues of $F$ as $\sigma$, we can obtain the Floquet exponents in the form

$$
\mu=\frac{1}{T} \ln \sigma .
$$

Thus, if we know the matrix $F$, the Floquet exponents are obtained from equation (17). In general, the matrix $F$ can be obtained by numerically integrating equation (12) over the period $T$. In the present study, the Crank-Nicolson method is employed for this process.

\section{Results and discussion}

\subsection{Basic features of the eigenvalue}

First, to confirm the features of the present system, the instantaneous amplification rate during the period drawn in figure 2 for the case of the TS wave-that is, the mode characterized by $(\alpha, \gamma)=(1.0,0.0)$-is plotted in figures 3 and 4 for various parameter values. The amplification rate is obtained as the imaginary part of the eigenvalue $\omega_{\mathrm{i}}$ of the Orr-Sommerfeld equation. If the sign of this value is positive, the disturbance will be amplified exponentially. The case estimated here is for $R_{e}=10000$, which corresponds to the supercritical condition (under the present definition, the critical Reynolds number is 5772). Figure 3 is obtained by $\Omega$ at 0.1 and $U_{\mathrm{w}}$ at each of $0.1,0.3,0.5$ and 0.7 . Figure 4 is obtained by $U_{\mathrm{w}}$ at 0.7 and $\Omega$ at each of $0.1,1.0,1.5$ and 2.0. The straight line plotted in these figures corresponds to the eigenvalue obtained by Orszag (1971) for the non-oscillating case. The sign of the eigenvalue changes periodically around zero. This means that the stability also alternates during the period of the wall oscillation, and it is not clear from this analysis whether the system is ultimately stable. Furthermore, these two figures show that the interval 


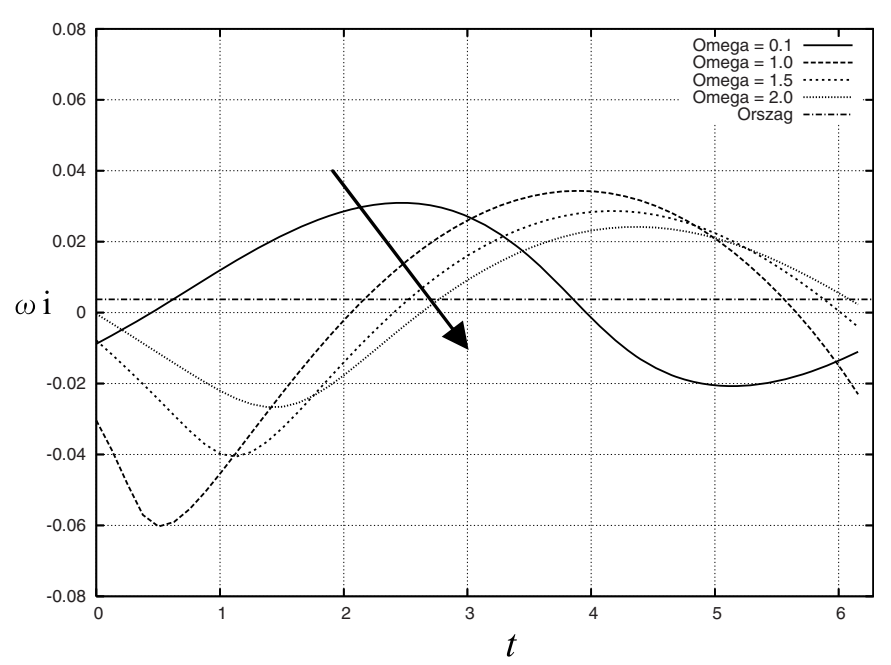

Figure 4. Variation of $\omega_{\mathrm{i}}$ during one period with $R_{e}=10000,(\alpha, \gamma)=(1,0), U_{\mathrm{w}}=0.7$ and $\Omega=0.1,0.5,1.5$ and 2.0 .

Table 1. Comparison of amplification rate of TS wave.

\begin{tabular}{ll}
\hline Floquet exponent $\mu$ (present) & 0.0037397 \\
\hline Imaginary part of $\omega_{\mathrm{i}}$ (Orszag 1971) & 0.0037396
\end{tabular}

of stability (or instability) cannot be determined from the phase of the wall oscillation alone. Thus, Floquet analysis is an effective means for analyzing such systems.

Before proceeding with Floquet analysis, the validity of the present approach is confirmed by comparison of the Floquet exponent $\mu$ obtained by equation (17) with the eigenvalues $\omega_{\mathrm{i}}$ of the usual Orr-Sommerfeld equation. Since the case $U_{\mathrm{w}}=0$ can be regarded as non-oscillating, the Floquet exponent $\mu$ should coincide with the eigenvalue given by Orszag (1971). Table 1 shows the result of comparing these values. These amplification rates agree well with each other.

\subsection{Stabilizing effects on $2 D$ disturbances}

Von Kerczek (Davis 1976) demonstrated stabilizing effects of wall oscillation when $U_{\mathrm{w}}$ is larger than some threshold. Figure 5 showed a $U_{\mathrm{w}}$ dependence of the stabilizing effect for some $R_{e}$, consistent with those results. The stabilizing effects seem to increase when $U_{\mathrm{w}}$ is increased. To understand this more deeply, $U_{\mathrm{w}}$ dependence is investigated for different frequencies with $R_{e}$ at 10000 ; the results obtained are shown in figure 6. Thus, wall oscillation does not universally stabilize. $\Omega$ dependence is also shown in figure 7 for some different values of $U_{\mathrm{w}}$. The stabilizing effect changes as $\Omega$ changes, and eventually destabilization occurs. Thus, it seems that the critical value of stability depends on the flow parameters $U_{\mathrm{w}}$ and $\Omega$. To provide a general view of this phenomenon, the contour of the Floquet exponent is shown in figure 8. The light region represents the area where the Floquet exponent is positive, which corresponds to the unstable region; the dark area represents negative Floquet exponents, which corresponds to the stable regions. The dash-dotted contours are drawn for steps of 0.002 in the 


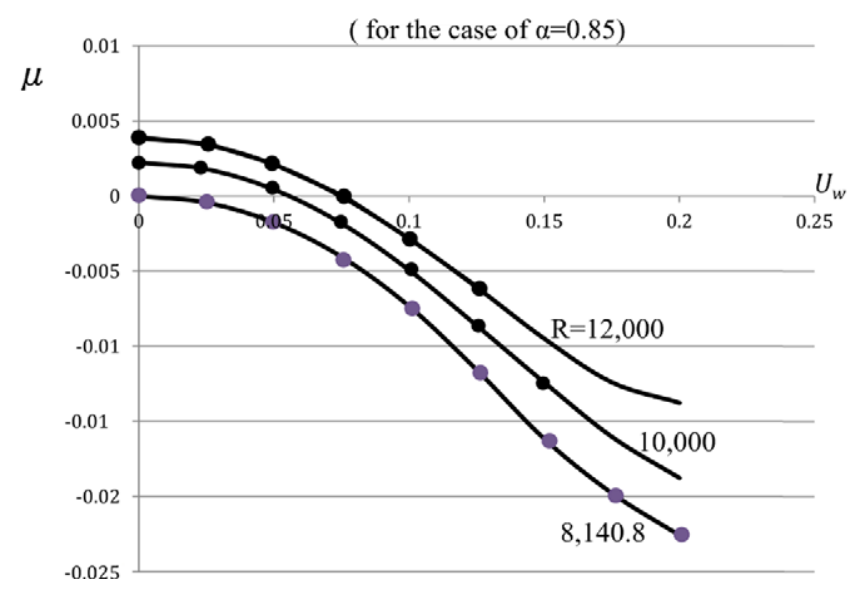

Figure 5. $U_{\mathrm{w}}$ dependence of the Floquet exponent for $(\alpha, \gamma)=(0.85,0)$ at some $R_{e}$, for comparison with Davis (1976).

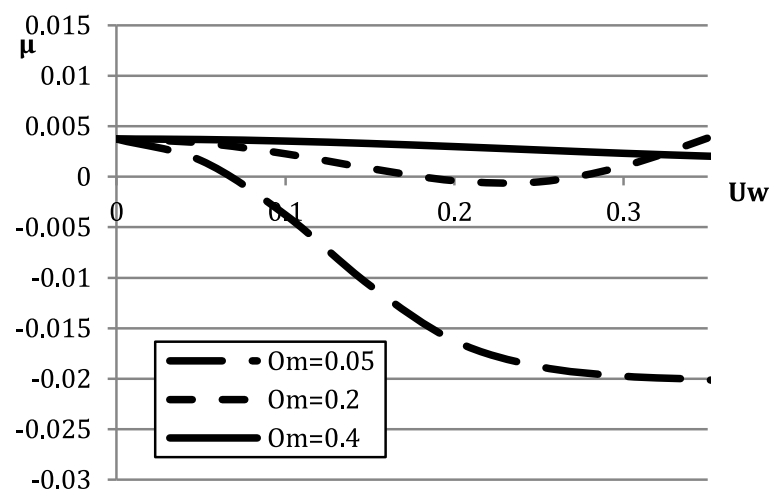

Figure 6. $U_{\mathrm{w}}$ dependence of the Floquet exponent for $R_{e}=10000,(\alpha, \gamma)=(1,0)$ and $\Omega=0.05,0.2$ and 0.4 .

Floquet exponent. A solid line in this figure represents the zero value of the Floquet exponent: the neutral curve. It is clear from the figure that the TS wave is stabilized depending on the value of the parameters even when the condition is supercritical. Furthermore, the valley of stable is deep. Thus, it is expected that the laminar-turbulent transition is delayed if the parameter values lie in the valley.

To examine the above features for other values of $R_{e}$, the $R_{e}$ dependence of the Floquet exponents are estimated and shown in figure 9. In most cases, the stabilizing effect increases as $R_{e}$ increases, but at some parameter values significant stabilizing effects are seen. The neutral curves for several $R_{e}$ are shown in figure 10 . When $R_{e}$ exceeds the critical value of 5772 , the stable region immediately appears. Although the width of the stable region decreases as $R_{e}$ increases beyond the critical value, it seems that the deep valley of stability along $\Omega$ near 0.05 does not disappear.

\subsection{Primary mode changing}

In this study, a 2D disturbance, specifically TS mode, has been investigated to show the basic aspects of the stabilizing effect on the present modified flow because it is well known from 


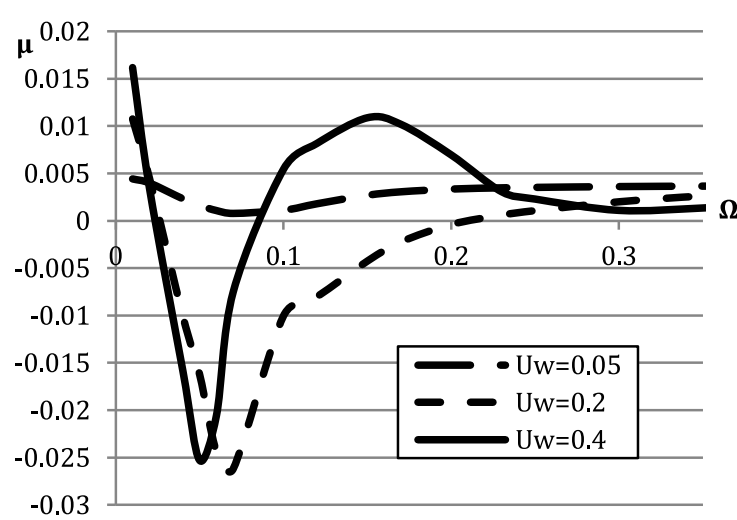

Figure 7. $\Omega$ dependence of the Floquet exponent for $R_{e}=10000,(\alpha, \gamma)=(1,0)$ and $U_{\mathrm{w}}=0.05,0.2$ and 0.4 .

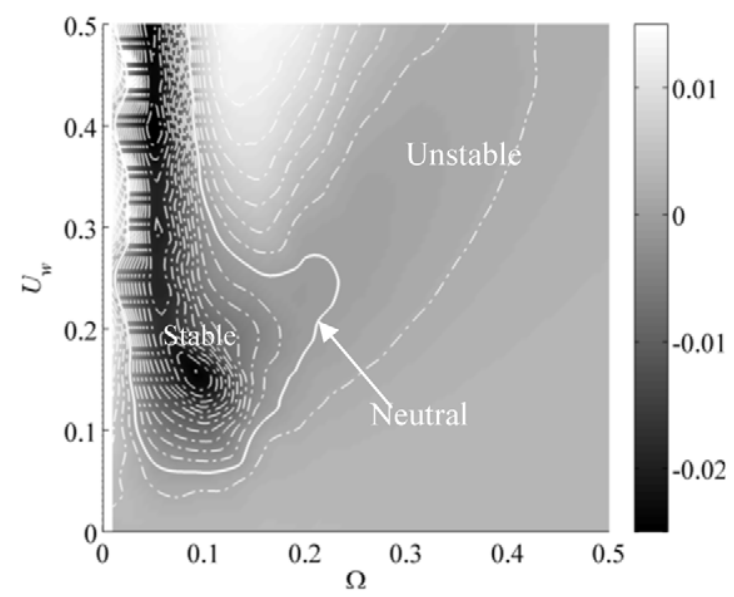

Figure 8. Contour of the Floquet exponent. The white area corresponds to the unstable region and the area black to the stable region. The solid line represents the neutral curve.

Squire's theorem that 2D disturbances show the most immediate effect. In this subsection, however, the stabilizing effect in oblique modes is investigated to develop a thorough understanding of this system. Tables $2-4$ show the Floquet exponents for the non-oscillating cases where $U_{\mathrm{w}}=0.0,\left(U_{\mathrm{w}}, \Omega\right)=(0.05,0.15)$ and $\left(U_{\mathrm{w}}, \Omega\right)=(0.1,0.15)$, respectively. In non-oscillating cases, TS mode shows the most unstable features, which is consistent with Squire's theorem. In contrast, for the oscillating case, when $U_{\mathrm{w}}$ increases the Floquet exponent of the TS wave decreases and finally the sign becomes negative. Although the Floquet exponents for the 3D mode also decrease as $U_{\mathrm{w}}$ increases, the ratio of decrease is relatively small. To understand this more deeply, the Floquet exponents of TS and oblique disturbances both with and without wall oscillation are shown in figure 11. The two upper lines correspond to the case without oscillation. When $R_{e}$ is not large, the TS wave of the Floquet exponent is large and the exponent first exceeds 0 . This means that the TS wave appears before the oblique wave. However, for the lower two lines, where oscillation is present, the value that 


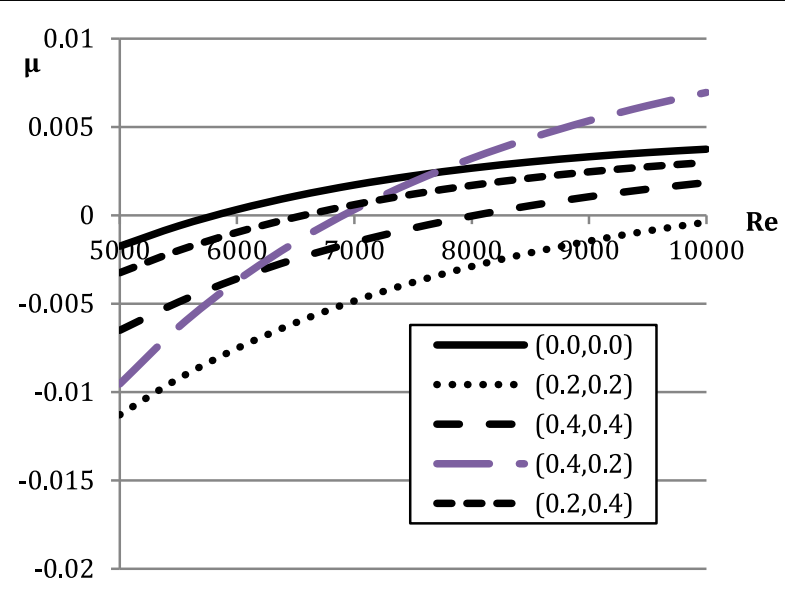

Figure 9. $R_{e}$ dependence of the Floquet exponent for $(\alpha, \gamma)=(1.0,0),\left(U_{\mathrm{w}}, \Omega\right)=$ $(0,0),(0.2,0.2),(0.4,0.4),(0.4,0.2)$ and $(0.2,0.2)$.

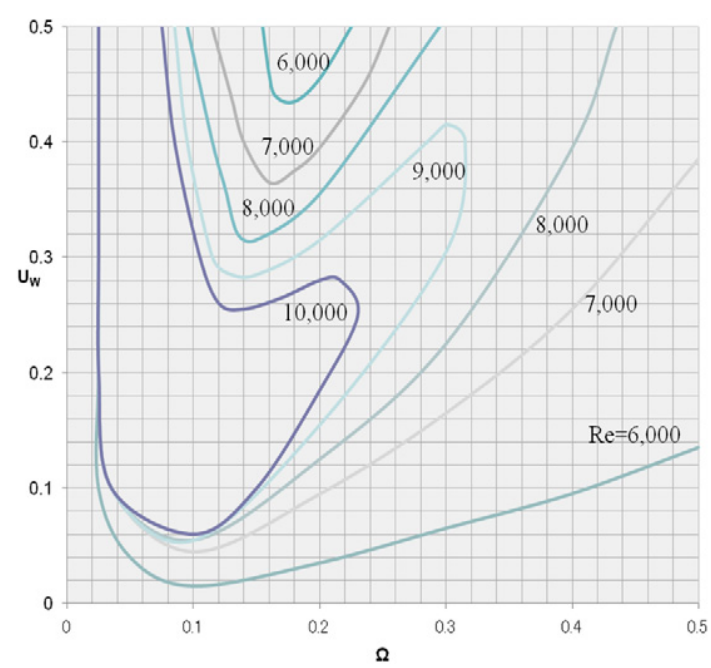

Figure 10. Neutral curves of the Floquet exponent for several $R_{e}$.

Table 2. The Floquet exponents for the case of non-oscillation and $R_{e}=10000$.

\begin{tabular}{llll}
\hline & $\alpha=1.0$ & $\alpha=0.9$ & $\alpha=0.8$ \\
\hline$\gamma=0.0$ & 0.00374 & 0.00362 & 0.00013 \\
$\gamma=0.1$ & 0.00361 & 0.00365 & 0.00034 \\
$\gamma=0.2$ & 0.00318 & 0.00370 & 0.00090 \\
$\gamma=0.3$ & 0.00234 & 0.00363 & 0.00162 \\
\hline
\end{tabular}

exceeds 0 value first is from the oblique wave. Consequently, wall oscillation makes oblique modes more unstable and induces primary mode changing. This result suggests that oblique waves can appear first in oscillating flow. 
Table 3. The Floquet exponents for the case $\left(U_{\mathrm{w}}, \Omega\right)=(0.05,0.15)$ and $R_{e}=10000$.

\begin{tabular}{lllr}
\hline & $\alpha=1.0$ & $\alpha=0.9$ & \multicolumn{1}{c}{$\alpha=0.8$} \\
\hline$\gamma=0.0$ & 0.00269 & 0.00285 & -0.00043 \\
$\gamma=0.1$ & 0.00256 & 0.00289 & -0.00022 \\
$\gamma=0.2$ & 0.00213 & 0.00295 & 0.00034 \\
$\gamma=0.3$ & 0.00130 & 0.00289 & 0.00108 \\
\hline
\end{tabular}

Table 4. The Floquet exponents for the case $\left(U_{\mathrm{w}}, \Omega\right)=(0.1,0.15)$ and $R_{e}=10000$.

\begin{tabular}{lllr}
\hline & $\alpha=1.0$ & $\alpha=0.9$ & \multicolumn{1}{c}{$\alpha=0.8$} \\
\hline$\gamma=0.0$ & -0.00018 & 0.00067 & -0.00209 \\
$\gamma=0.1$ & -00030 & 0.00072 & 0.00186 \\
$\gamma=0.2$ & -0.00071 & 0.00081 & -0.00126 \\
$\gamma=0.3$ & -0.00151 & 0.00081 & -0.00046 \\
\hline
\end{tabular}

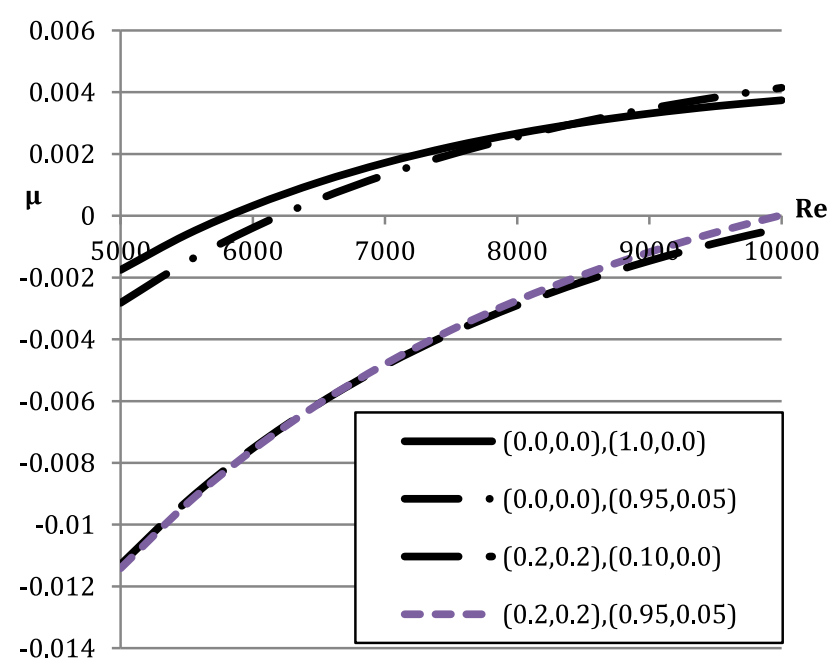

Figure 11. $R_{e}$ dependence of the Floquet exponent for $(\alpha, \gamma)=(1,0),(0.95,0.05)$ and $\left(U_{\mathrm{w}}, \Omega\right)=(0,0),(0.2,0.2)$.

\section{Conclusion}

The effect of longitudinal wall oscillation on 2D channel flow was analytically investigated. Because this system has time periodicity from wall oscillation, Floquet theory was employed. Thus, stability was described by the Floquet exponents. For the Floquet analysis, a monodromy matrix was derived from a time-dependent Orr-Sommerfeld equation using the collocation method. The governing parameters of this system were the frequency and amplitude of wall oscillations and the Reynolds number which is determined by maximum velocity of mean flow and half the distance between two walls. For Reynolds numbers in the range 6000-10000, the TS mode became stable depending on the parameters even when the system was under the supercritical condition. These parameters formed a deep valley stable region on the parameter space. Furthermore, it was shown that an increase in the amplitude 
of wall oscillation decreased the stabilizing effect, especially for 2D TS mode. Thus, 3D mode also became more unstable. Consequently, primary mode changing occurred, contrary to Squire's theorem.

\section{References}

Blennerhassett P J and Bassom A P 2002 The linear stability of flat stokes layers J. Fluid Mech. $464393-410$

Canuto C, Hussaini M Y, Quarteroni A and Zang T A 1988 Spectral Methods in Fluid Dynamics (Berlin: Springer)

Choi K-S 2002 Near-wall structure of turbulent boundary layer with spanwise-wall oscillation Phys. Fluids 14 2530-42

Choi H, Moin P and Kim J 1994 Active turbulence control for drag reduction in wall bounded flows J. Fluid Mech. 262 75-110

Davis S H 1976 The stability of time-periodic flows Annu. Rev. Fluid Mech. 8 57-74

Floryan J M 1997 Stability of wall-bounded shear layers in the presence of simulated distributed surface roughness J. Fluid Mech. 335 29-55

Floryan J M, Szumbarski J and Wu X 2002 Stability of a flow in a channel with vibrating walls Phys. Fluids 14 3927-36

Grosch C E and Salwen H 1968 The stability of steady and time-dependent plane Poiseuille flow J. Fluid Mech. 34 177-205

Hall P 1975 The stability of the Poseuille flow modulated at high frequencies Proc. R. Soc. Lond. A344 453-64

Herbert D M 1972 The energy balance in modulated plane Poiseuille flow J. Fluid Mech. 56 73-80

Jovanovic M R 2008 Turbulent suppression in channel flows by small amplitude transverse wall oscillation Phys. Fluids 20014101

Jung W J, Mangiavacchi N and Akhavan R 1992 Suppression of turbulence in wall-bounded flows by high-frequency spanwise oscillations Phys. Fluids 4 1605-7

Kim J, Moin P and Moser R 1987 Turbulence statistics in fully developed channel flow at low Reynolds number J. Fluid Mech. 177 133-66

Orszag S A 1971 Accurate solution of the Orr-Sommerfeld stability equation J. Fluid Mech. 50689 1441-7

Quadrio M and Ricco P 2004 Critical assessment of turbulent drag reduction through spanwise wall oscillation J. Fluid Mech. 521 251-71

Ricco P and Quadrio M 2008 Wall-oscillation conditions for drag reduction in turbulent channel flow Int. J. Heat Fluid Flow 29 891-902

Selvarajan S, Tulapurkara E G and Vasanta Ram V 1999 Stability characteristics of wavy walled channel flows Phys. Fluids 11 579-89

Sumitani Y and Kasagi N 1995 Direct numerical simulation of turbulent transport with uniform wall injection and suction AIAA J. 33 1220-8

Thomas C, Bassom A P and Blennerhassett P J 2011 The linear stability of oscillatory Poseuille flow in channels and pipes Proc. R. Soc. Lond. A467 2643-62

Von Kerczek C P 1982 The instability of oscillation plane Poiseuille flow J. Fluid Mech. 116 91-114

Zamir M 2000 The Physics of Pulsatile Flow (New York: AIP Press/Springer) 